\title{
Echocardiographic guidance for transcatheter mitral valve repair using edge-to-edge clip
}

\author{
Edris Aman ${ }^{1} \cdot$ Thomas W. Smith $^{1}$ (1)
}

Received: 29 November 2018 / Accepted: 18 January 2019 / Published online: 15 February 2019

(c) The Author(s) 2019

\begin{abstract}
Percutaneous intervention for mitral valve disease has been established as an alternative to open surgical repair in high risk and inoperable candidates. Edge-to-edge leaflet plication with Mitraclip (Abbott, Menlo Park, CA, USA) is indicated for primary and secondary mitral valve diseases. Echocardiography provides a better understanding of mitral valve anatomy and allows us to classify and quantify mitral regurgitation. Transesophageal echocardiography is essential in patient screening, intraprocedural guidance, and post-procedure evaluation for patients undergoing edge-to-edge plication with MitraClip.
\end{abstract}

Keywords Mitral regurgitation · Transcatheter mitral valve repair · Interventional echocardiography · Three-dimensional transesophageal echocardiography $\cdot$ Valvular heart disease

\section{Introduction}

Percutaneous intervention for mitral valve disease has been established as an alternative to open surgical repair, especially in high risk and inoperable candidates [1]. Edge-toedge leaflet plication with the MitraClip (Abbott, Menlo Park, California, USA) is indicated in Japan for primary or secondary mitral valve disease. Studies have underlined the therapeutic benefit of MitraClip for patients with primary mitral valve disease, particularly an improvement in functional capacity and quality of life [2]. Recently, the COAPT trial demonstrated a reduction in hospitalizations and improved mortality with edge-to-edge clip in patients on optimal heart failure therapy with significant functional mitral regurgitation [3]. The MitraClip procedure shows low periprocedural risk and a significant reduction in mitral valve regurgitation. During the procedure, the anterior and posterior leaflets are "clipped;" analogous to the Alfieri surgical technique [4]. Transesophageal echocardiography is essential to procedural success: being utilized in the pre-procedure evaluation, procedural guidance, and post-procedure evaluation [5].

Thomas W. Smith

twrsmith@ucdavis.edu

1 Division of Cardiology, Department of Internal Medicine, University of California, Davis, 4860 Y Street, Suite 2820, Sacramento, CA 95817, USA

\section{Mitral valve anatomy}

The mitral valve apparatus is complex and includes the mitral annulus, anterior and posterior leaflets, chordae tendinea and papillary muscles. The left ventricular wall plays a key role in functional MR although is not a defined component of the apparatus. The mitral annulus itself includes the aortic annulus, anterior mitral leaflet, annuloaorta fibrosa and tricuspid annulus. Anterior and posterior leaflets of the mitral valve are generally defined with a sixscallop structure with various nomenclature definitions. The posterior leaflet generally has 3 scallops-the most lateral (adjacent to the left atrial appendage) is P1 with P3 being the most medial scallop (adjacent to the interatrial septum). The middle scallop is P2. As the anterior leaflet does not have scallops, the anterior leaflet location is defined by the adjacent posterior scallop. For example, A1 is immediately across from P1. Anatomical evaluation of the mitral valve utilizing transesophageal echocardiography (TEE) is critical for edge-to-edge procedural success.

Alterations in any part of the mitral valve apparatus or the left ventricle can affect the coaptation between the anterior and posterior leaflets and lead to significant mitral regurgitation. Broadly, coaptation between the anterior and posterior leaflets may be compromised by annular dilation, damaged, flail or prolapsed leaflets, leaflet thickening, chordae restriction, and/or left ventricular abnormalities (Fig. 1). There are many etiologies of mitral valve disease; these include 


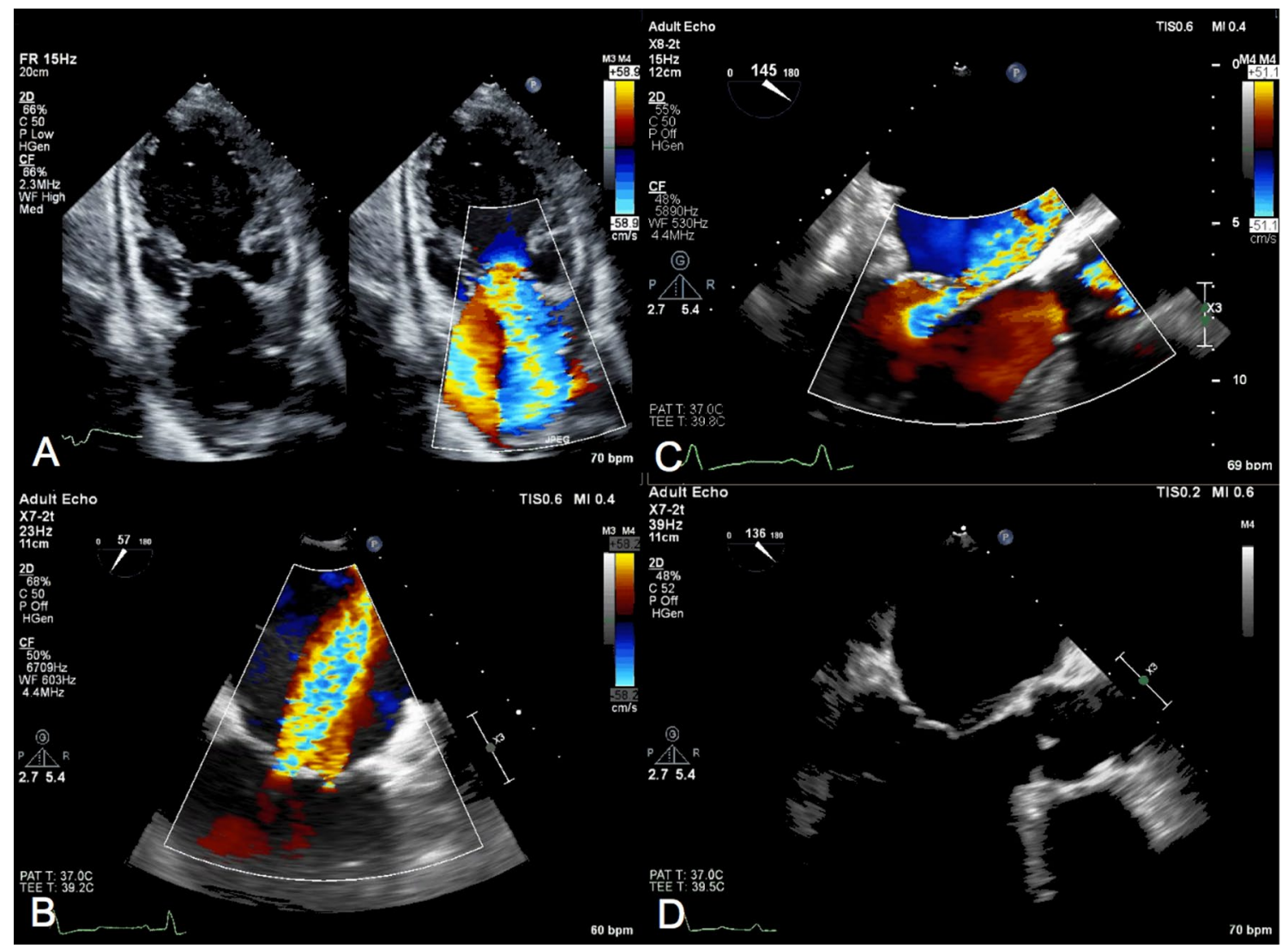

Fig. 1 Mitral valve disease is variable. Mitral valve regurgitation may be secondary to a broad range of disease processes from primary mitral valve disease involving the mitral apparatus to secondary mitral valve disease due to left ventricular dysfunction. a Severe functional mitral regurgitation with restricted and thickened leaflets.

fibroelastic deficiency, Barlows, rheumatic mitral valve disease, congenital mitral cleft, calcified annulus, infective endocarditis, marantic endocarditis, coronary artery disease, dilated cardiomyopathy, etc. Each of these disease processes can uniquely affect the valve apparatus with variable damage to each component.

\section{Preprocedural transthoracic imaging}

For percutaneous mitral valve interventions, TEE is the primary imaging modality for qualitative assessment of mitral regurgitation and procedural guidance. In preprocedure, transthoracic echocardiography (TTE) is a robust tool to identify mitral valve disease, quantify its severity and help distinguish the etiology [6] (Fig. 2).

The parasternal long-axis view allows visualization of the middle scallops (A2/P2) and assessment for thickening, calcification, degenerative changes and malcoaptation. Visual qualitative estimation of the mitral regurgitation jet and its physical characterization, specifically direction, can b Malcoaptation with degenerative changes of A2/P2 scallops. c Posterior leaflet flail with resultant anteriorly directed mitral regurgitation. $\mathbf{d}$ Left atrial enlargement and prior inferior myocardial infarction resulting in restricted leaflets and malcoaptation

help elucidate the etiology. Additional views are necessary to evaluate for non-central regurgitant jets and to appropriately quantify the severity of regurgitation.

The apical four-chamber view provides quantitative assessment of mitral regurgitation by proximal isovelocity surface area derived effective regurgitation orifice area. Measurement of transvalvular gradient and calculation of mitral valve area can be assessed in this view. Primary mitral valve pathologies such as restriction, thickening, prolapse, and flail can also be appreciated in the apical four-chamber view.

In the two-chamber view, the scan view is parallel to the line of coaptation and thus the entire width of the mitral regurgitation jet, from medial to lateral commissure, is assessed. A very broad jet in this view may indicate the need for multiple clips in order to successfully approximate leaflets and increase coaptation.

Lastly, the parasternal short axis allows mitral valve area planimetry at the leaflet tips. This provides a direct measurement of the diastolic mitral valve area that is independent of loading conditions. 


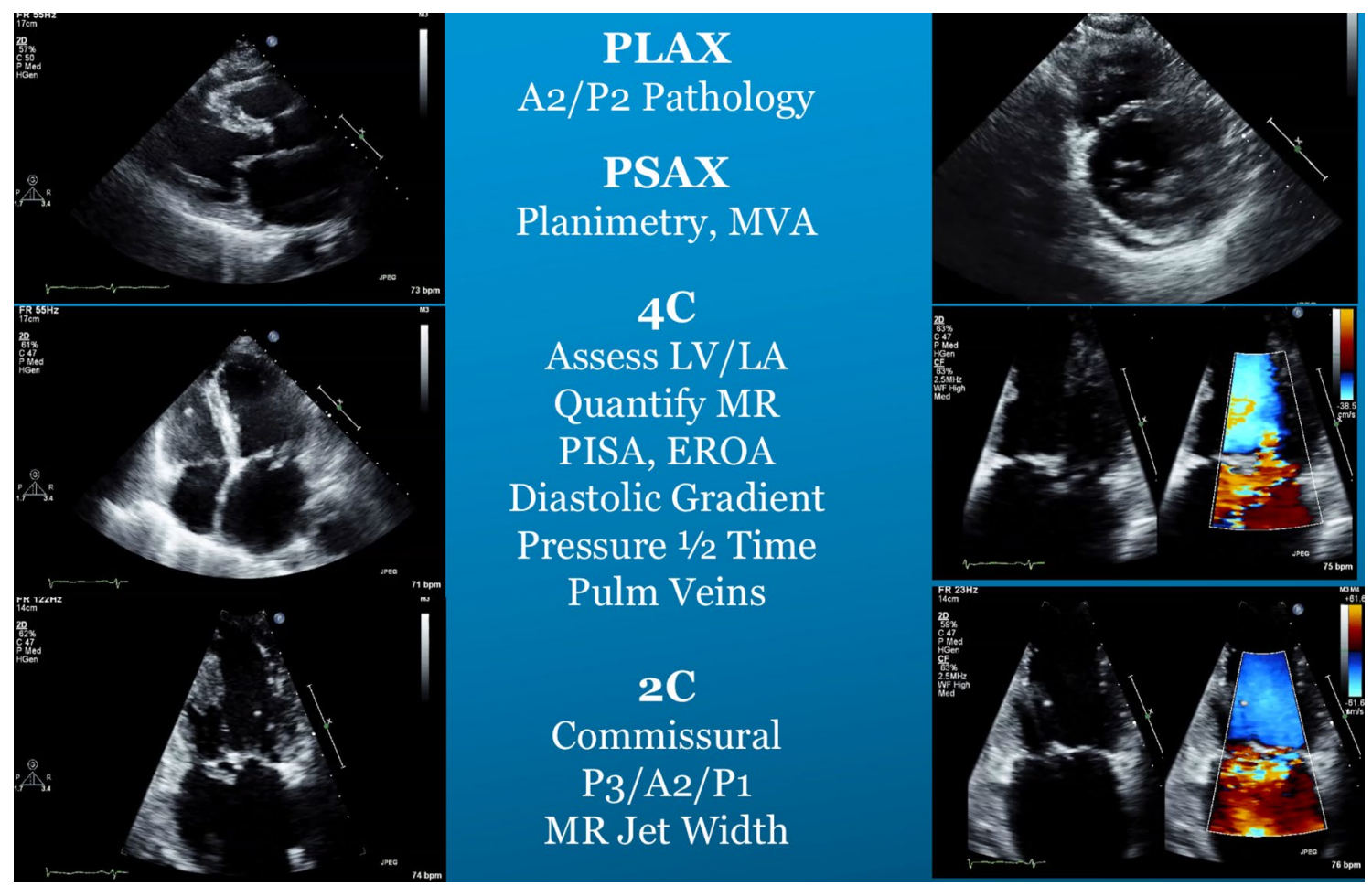

Fig. 2 Transthoracic imaging views. TTE is a key tool in the evaluation of mitral regurgitation. A thorough TTE evaluation provides MR severity assessment and often is sufficient in establishing the structural abnormality leading to the MR

\section{Preprocedural and intraprocedural transesophageal echocardiogram exam}

Screening TEE allows for a thorough evaluation of the mitral valve apparatus to pinpoint the etiology of mitral regurgitation and predict feasibility and safety of transcatheter mitral valve repair. The basic imaging strategy using TEE involves sweeping the entire valve from three different orientations. In addition to $2 \mathrm{D}$ and $3 \mathrm{D}$ visual assessment, Doppler interrogation will provide quantification of mitral regurgitation and mitral valve area and gradients. Interrogation of the interatrial septum, left atrial appendage and pulmonary veins are also critical. Through a thorough investigation of the mitral valve, the echocardiographer should develop their own comprehensive three-dimensional model and recognize the probe positions necessary for procedural guidance. TEEs are generally performed following a TTE to provide the operator with an assessment of the severity of MR, likelihood of success and identification of possible procedural complications. Patients generally have a TEE in the initial evaluation and then confirmatory images are taken immediately prior to the invasive procedure.

With omniplane set at $0^{\circ}$, the lateral (A1/P1) to the medial (A3/P3) aspect of the valve is evaluated by advancing the probe a few centimeters in the esophagus (Fig. 3). Additionally, applying probe anteroflexion and then retroflexion in the mid-esophageal view will also sweep lateral to medial along the valve.

The intercommissural view is defined by the omniplane angle and mid-esophageal probe position that provides visualization of the P1/A2/P3 mitral scallops (Figs. 4, 5). Generally, this view is obtained with an omniplane angle of $45^{\circ}-60^{\circ}$. Clockwise and counterclockwise rotation of the probe will visualize the anterior leaflet and posterior leaflets, respectively, in a plane parallel with the coaptation line formed between A2:P2.

The long axis, typically $135^{\circ}-150^{\circ}$, allows evaluation of A2/P2 mitral scallops at the long axis of the aortic valve. From this position, rotation of the probe counterclockwise will sweep lateral toward the LAA and clockwise rotation will move medially toward the interatrial septum (Fig. 6). If valve pathology is present at $\mathrm{A} 2 / \mathrm{P} 2$, then this view will provide an approximate grasping view as it is perpendicular to the coaptation line between the A2 and P2 scallops.

In addition to sweeping through the mitral valve in the above omniplane orientations in $2 \mathrm{D}, 3 \mathrm{D}$ en face imaging provides additional information and creates a relatively comprehensive view of the MV from the atrial perspective to aid in planning and communication with the implant team. 3D should be used in conjunction with 2D, but never instead of 2D (Fig. 7). Due to its lower frame rate and limited subvalvular assessment, 3D TEE may miss subtle MV apparatus 


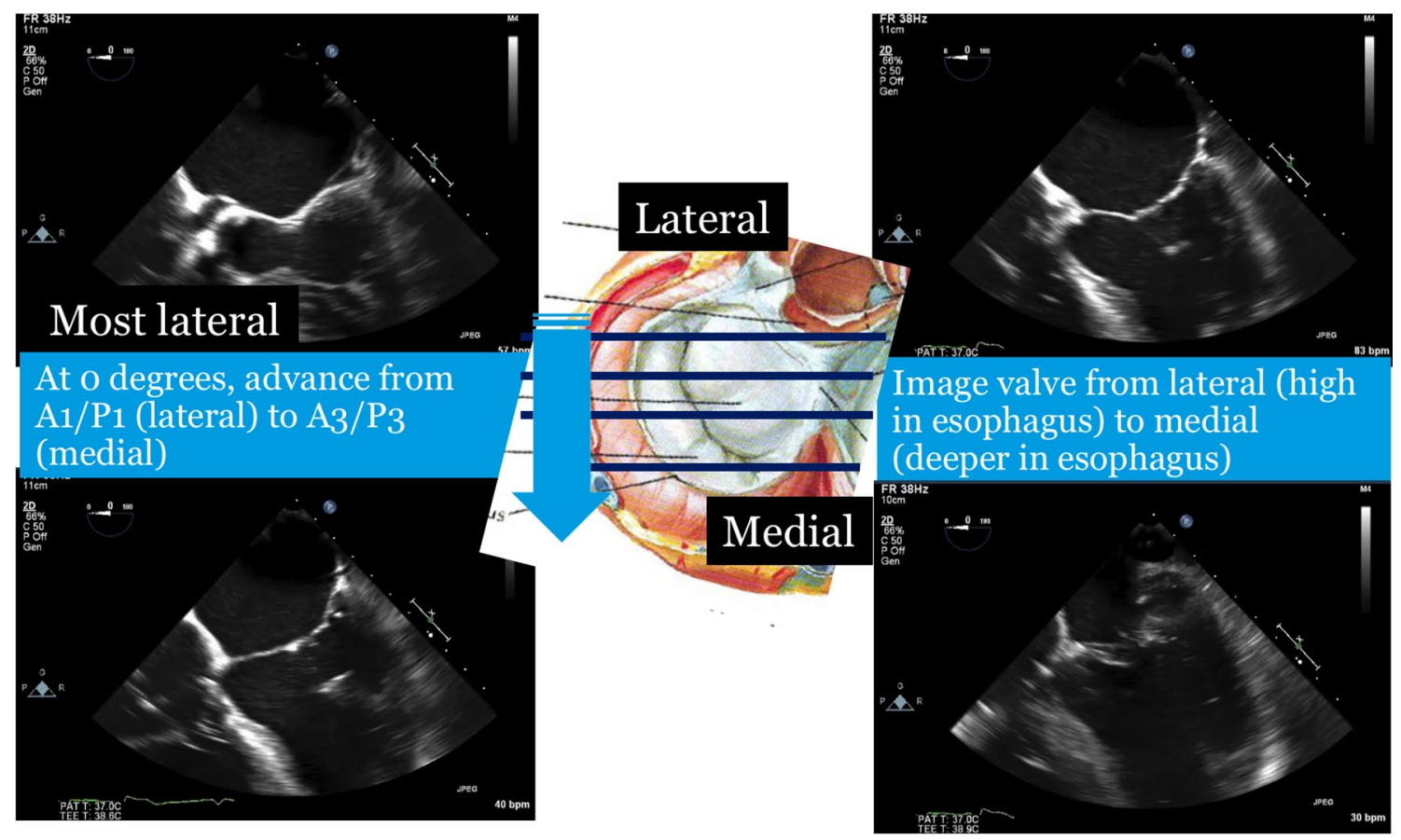

Fig. 3 Sweep at $0^{\circ}$. Advancing the probe from a high mid-esophageal position at $0^{\circ}$ to a low mid-esophageal position will sweep from the lateral aspect of the valve (A1/P1) to the medial aspect (A3/P3)

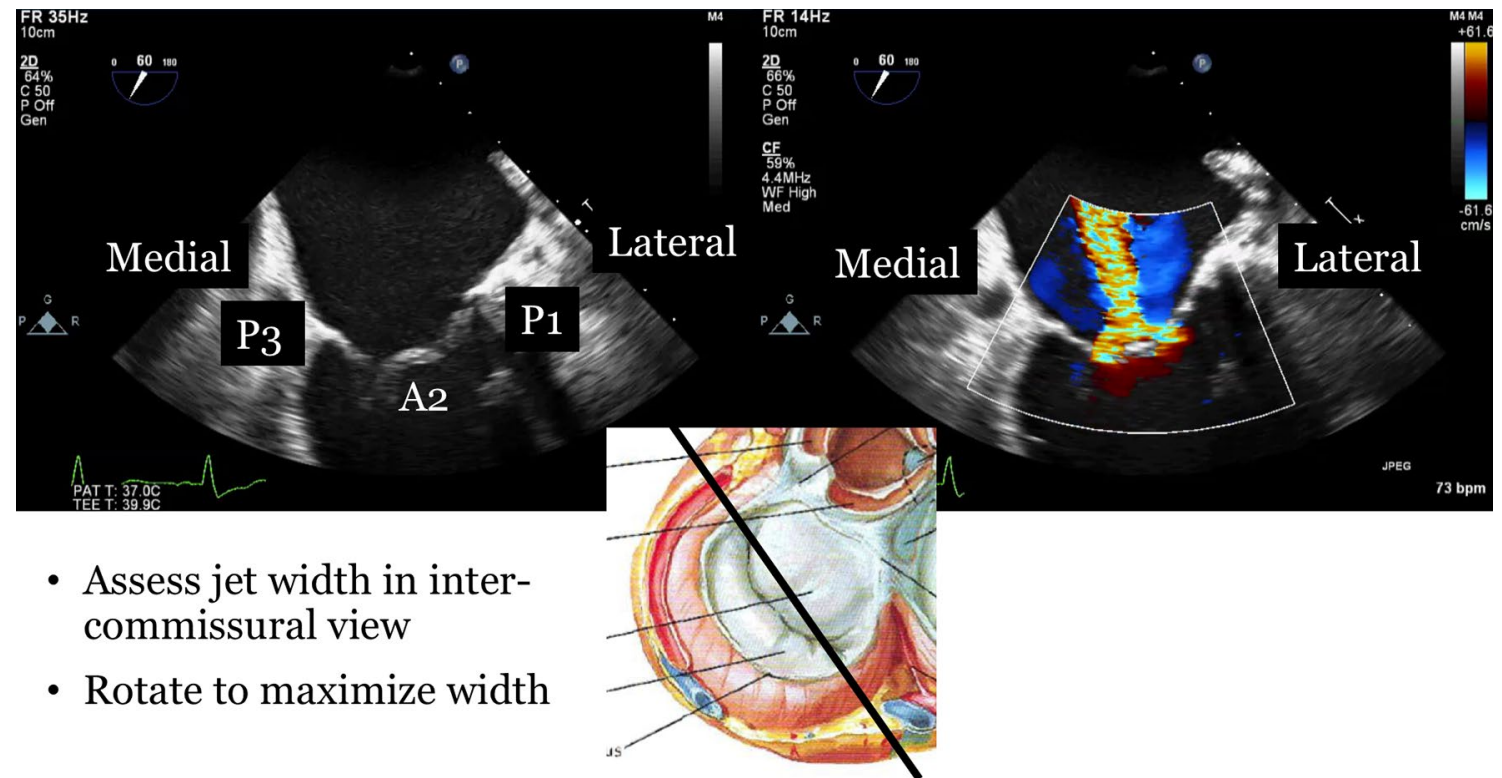

Fig. 4 Intercommissural view. Moving the omnioplane to the angle necessary to visualize P3/A2/P1 aligns the imaging plane parallel to the coaptation line at $\mathrm{A} 2 / \mathrm{P} 2$. It allows visualization of central mitral

abnormalities. Notably, leaflet abnormalities such as deep scallops or clefts may be more apparent on 3D than 2D.

The interatrial septum, left atrial appendage, and pulmonary veins are subsequently visualized. Knowledge and visualization of the interatrial septum anatomy are regurgitation and an assessment of the width of the jet in the medial/ lateral aspect

necessary to safely guide TEE, the transseptal puncture. The presence of an interatrial septal aneurysm, lipomatous septum, atrial septal defect or patent foramen ovale should be noted. In the rare patient where the interatrial septum is not well visualized with TEE, an alternative 


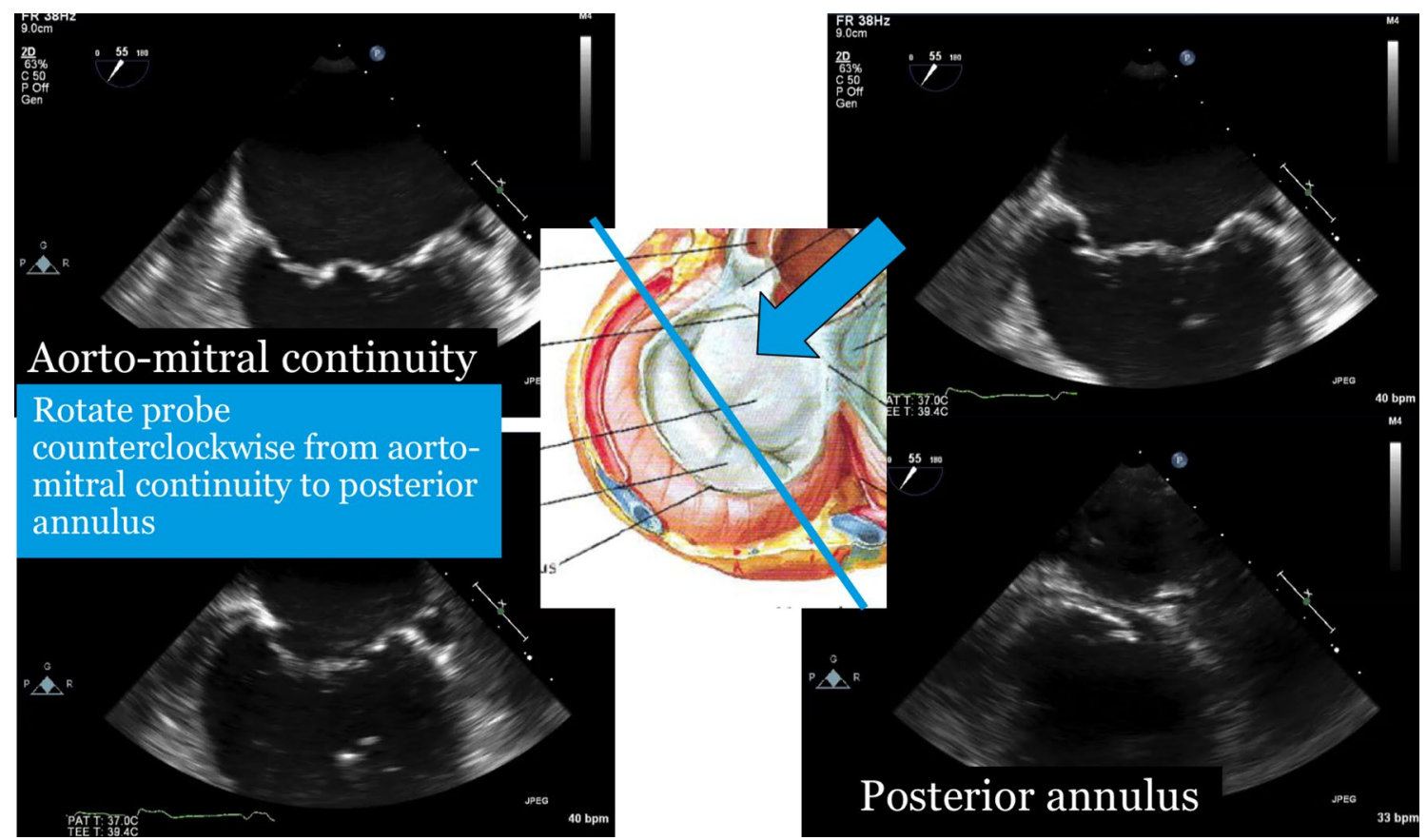

Fig. 5 Intercommissural sweep. From the intercommissural view parallel to A2/P2 coaptation line, a clockwise rotation of the TEE probe will allow visualization of the aortomitral continuity. From the aorto- mitral continuity, a counterclockwise rotation will move the imaging plane back through $\mathrm{P} 3 / \mathrm{A} 2 / \mathrm{P} 1$ and then to the posterior leaflet and posterior annulus

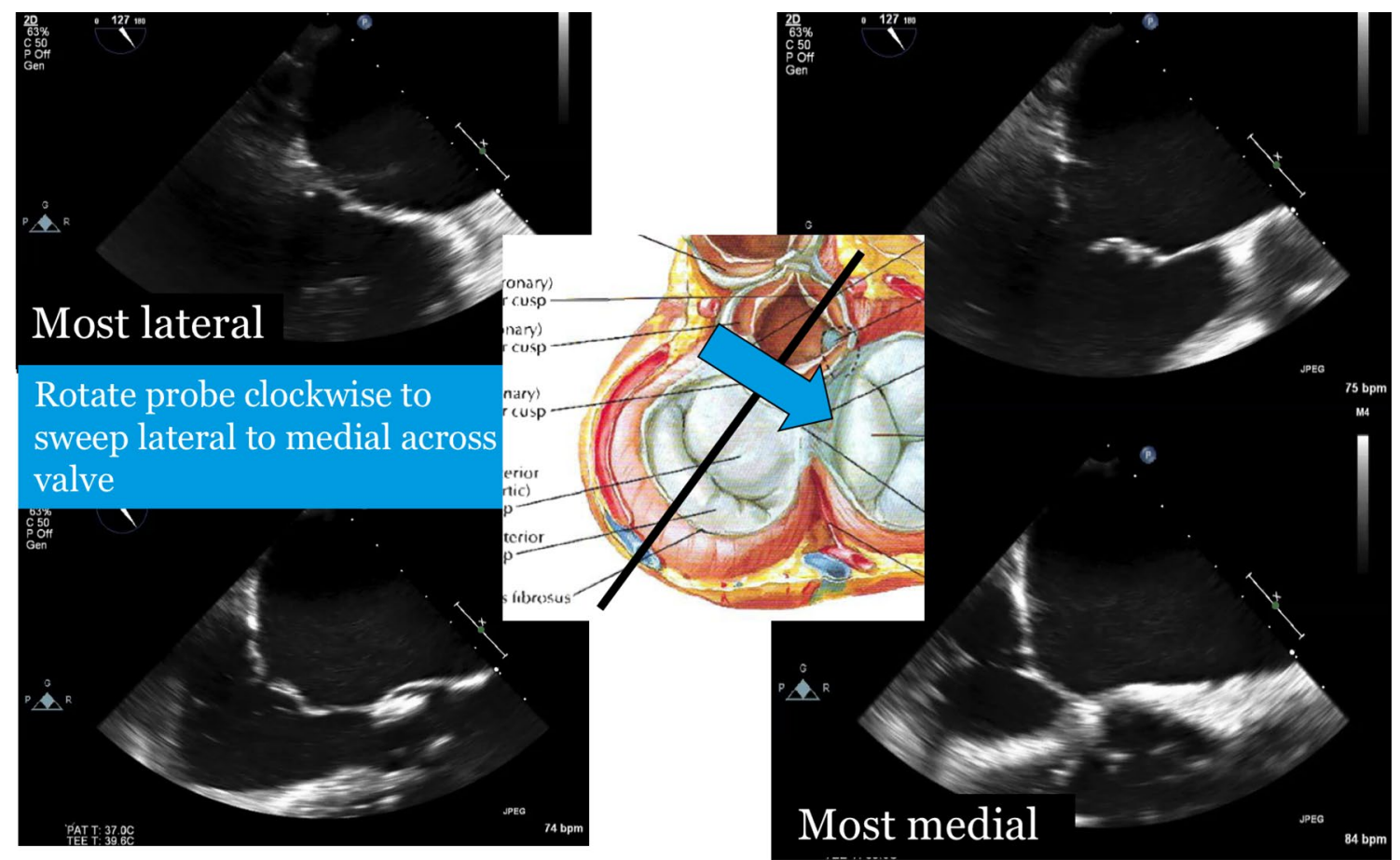

Fig. 6 Long-axis sweep. The TEE omniplane is adjusted to demonstrate the long-axis view of the aortic valve and the A2/P2 scallops of the mitral valve. This view is perpendicular to the line of coaptation at A2/P2. With counterclockwise rotation, the TEE imaging plane moves laterally toward A1/P1 and with clockwise rotation, it moves medially toward A3/P3 


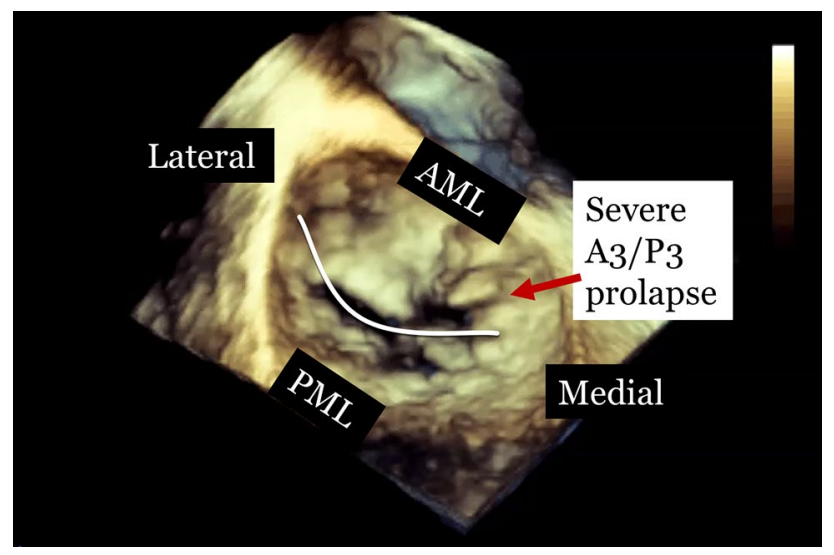

Fig. 7 3D En face view of the mitral valve demonstrating severe medial degenerative pathology. A 3D view of the mitral valve from the left atrial perspective demonstrates the majority of the mitral leaflet pathology and assists in summarizing the mitral valve findings from the prior sweeps. Lateral lateral valve, A1/P1 side, Medial medial valve, A3/P3 side, AML anterior mitral leaflet, PML posterior mitral leaflet, White line coaptation line between the anterior and posterior leaflets

imaging modality such as Intracardiac Echocardiography (ICE) may be necessary. Thrombus should be excluded from the left atrial appendage and both atria. The pulmonary veins are utilized to assist in characterization of severity of mitral regurgitation and later to determine if there is improvement in mitral regurgitation post-edge to edge plication. Assess at least one right and one left pulmonary vein to exclude artifact from an eccentric jet entering the respective vein in systole. A transgastric view is also helpful in assessing the short-axis view of the mitral valve as well as LV function and the presence of any pre-procedure pericardial effusion. While the transgastric view had been critical in aligning the grasp, since the advent of quality real-time 3D imaging it has not been necessary.

\section{Procedural image guidance with TEE}

After confirming prior valve findings, TEE is instrumental in guiding the MitraClip procedure. Procedural steps include transseptal puncture, advancement of steerable guide catheter (SGC) into the left atrium, insertion of the clip delivery system (CDS) through the SGC into the left atrium, positioning the clip above the grasping area, advancing the clip into the left ventricle, grasping the leaflets, comprehensive valve assessment, clip release and removal of the CDS. If another clip is necessary, an additional CDS is advanced through the SGC.

\section{Transseptal puncture}

Understanding the anatomy of the interatrial septum and corresponding TEE views will optimize accurate transseptal puncture. An optimal transseptal puncture site is important to establish adequate height above the mitral valve pathology.

Three 2D TEE planes are primary used to define the puncture site. The initial view is the bicaval view at $90^{\circ}-120^{\circ}$ which allows superior-inferior orientation. Subsequently, a short-axis view at the base $\left(30^{\circ}-50^{\circ}\right)$ allows for anterior-posterior orientation (Fig. 8) The third view is a four-chamber view at $0^{\circ}$ to determine height above the mitral valve.

The transseptal puncture starts after the interventionalists pass a wire and catheter into the superior vena cava (SVC). The catheter is then withdrawn into the left atrium, along the interatrial septum and the position of the tip of the transseptal needle can be identified by a tent-like dooming of the interatrial septum towards the left atrium. In general, a midto-superior fossa position is favored. Once the superior-inferior orientation within the fossa has been confirmed in the bicaval view, the short-axis view can be utilized to evaluate the anterior-posterior position. The catheter may be rotated anteriorly toward the aortic valve or posteriorly toward the atrial posterior wall. A posterior position will place the catheter higher above the mitral annulus. Generally, a posterior and mid-to-superior puncture site is recommended. Once in this initial position, a four-chamber view is utilized to determine how high the crossing point is above the mitral valve.

The optimal puncture height depends on the height of the mitral valve pathology. A valve with primary (degenerative) mitral regurgitation may have a coaptation point above the annulus while a patient with secondary (functional) mitral regurgitation may have a coaptation point below the annulus. A height $4.5 \mathrm{~cm}$ above the valve pathology is a reasonable height to target. As the catheter gains height relative to the annulus as it moves laterally across the atrium, medial pathology may require an even higher crossing point while lateral pathology may be successful with a lower crossing height. Access via a patent foramen ovale is not recommended as the defect is generally too anterior and the tunnel constrains the trajectory of the SGC.

Crossing the interatrial septum should be visualized in the short axis at the base view. This allows imaging of the distal portion of the transseptal needle as it transitions into the left atrium. With adequate visualization of the path of the needle, atrial injury may be avoided. Additional imaging techniques such as biplane or 3D imaging of the transseptal step may be used although it remains imperative to see the needle trajectory and avoid the atrial wall. After successful transseptal, a catheter is visualized advancing into the left atrium and an exchange length guidewire is preferentially 


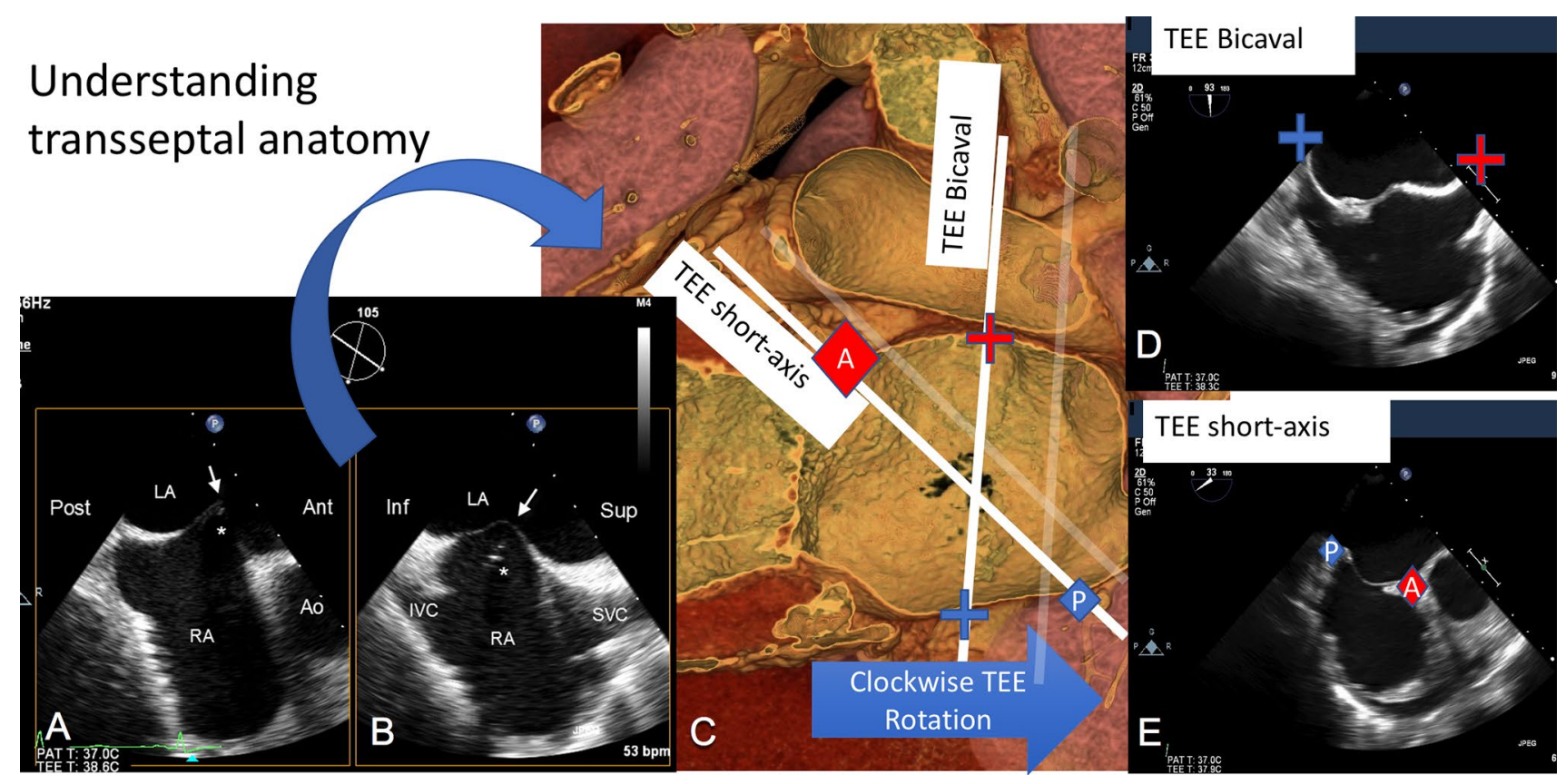

Fig. 8 Transseptal anatomy and puncture. a Short-axis at the base demonstrating anterior and posterior orientations of the interatrial septum. b Bicaval view of the interatrial septum demonstrating superior and inferior orientations. c Perspective of interatrial septum from the left atrium. TEE short-axis plane and Bicaval plane are demonstrated with solid white lines. The transparent white lines show how

placed in the left superior pulmonary vein (LUPV). With a counterclockwise rotation from the short axis at the base view, the TEE can guide the wire into the LUPV, avoiding any potential injury to the left atrial appendage.

\section{Steerable guide catheter (SGC) placement into the left atrium}

Following placement of the exchange length guidewire into the left upper pulmonary vein, the SGC and dilator assembly are gently advanced into the left atrium. The dilator can be identified by an echogenic appearance of striations at the cone tip. The tip of the SGC is marked with a radiopaque, echo-bright double ring. The insertion of the SGC should be carefully monitored with TEE and fluoroscopic imaging to avoid injury to the atrial wall (Fig. 9). At the transition between the dilator and the guide catheter, there may be a small increase in catheter size. This can occasionally cause the interatrial septum to tent at the transition point. Continuous imaging with an omniplane that demonstrates the entire dilator tip, transition, guide catheter tip and interatrial septum will provide the interventionalist with the imaging information to safely and slowly advance the SGC into the left atrium. Once the SGC is $2-3 \mathrm{~cm}$ into the left atrium, the wire and dilator are removed. the planes change with a clockwise rotation of the TEE probe-moving further away from the mitral valve annulus. d Bicaval view. e Short-axis at the base view. Post posterior, $L A$ left atrium, $R A$ right atrium, $A n t$ anterior, $I V C$ inferior vena cava, $S V C$ superior vena cava, Red $A$ anterior, Blue $P$ posterior, Red + superior septum, Blue + inferior septum

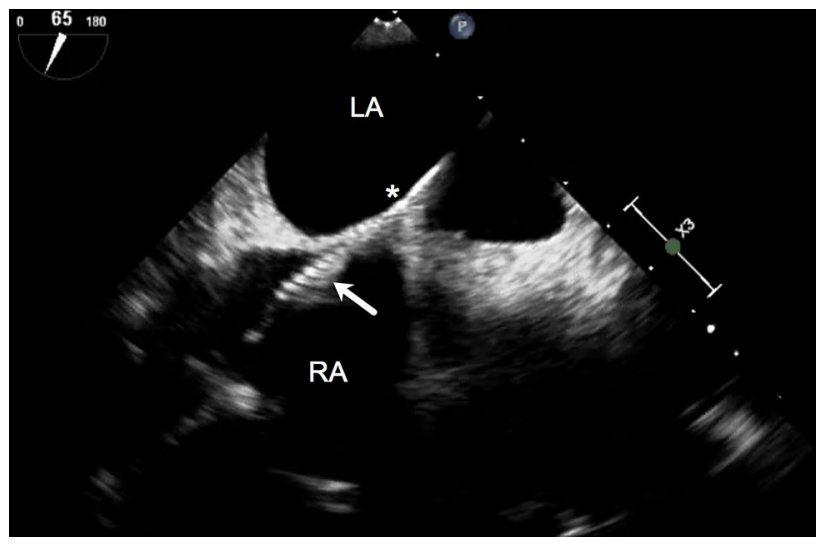

Fig. 9 Steerable guide catheter (SGC) with dilator crossing interatrial septum. The echogenic coils are visualized at the distal end of the dilator (arrow) as it crosses and tents the interatrial septum (asterisk). TEE should approximate the trajectory and anticipated travel of the $\mathrm{SGC}$ as it enters the left atrium. $L A$ left atrium, $R A$ right atrium

\section{Clip delivery system (CDS)}

The CDS is advanced through the SGC until the clip is at the tip of the SGC; this is visualized under fluoroscopic guidance. Saline flush is visualized prior to CDS reaching the tip of the SGC. The CDS is then advanced into the left atrium under TEE and fluoroscopic guidance. The CDS is tracked 
across the left atrium as it travels laterally toward the lateral left atrial wall, left upper pulmonary vein and left atrial appendage. The TEE manipulation is similar to tracking the initial wire across the left atrium, counterclockwise rotation and slight withdrawal of the TEE with an omniplane in the $35^{\circ}-55^{\circ}$ range. As the CDS approaches the left atrial wall, it is imperative to visualize both the distal CDS and the atrial wall to allow the interventionalist to manipulate the CDS position without atrial wall injury. As the CDS is moved more medial across the aortomitral continuity and posteriorly to a position above the middle of the mitral valve, the TEE may be advanced and rotated to approach an intercommissural view. Although 3D will visualize most the left atrium and the CDS, it lacks the resolution that 2D has when the CDS is directly adjacent to the lateral atrial wall-where the CDS may be millimeters away from the wall. For this reason, we prefer to steer down to the valve with $2 \mathrm{D}$ and reserve $3 \mathrm{D}$ for cases where gross CDS movements in the center of the LA are necessary, such as steering away from the aorta.

\section{MitraClip positioning in the left atrium}

After the CDS is positioned above the mitral valve, biplane imaging provides an efficient method for the interventionalist to position the CDS in the appropriate position above the valve. Two orthogonal TEE planes are involved in clip positioning, a mid-esophageal intercommissural view $\left(40^{\circ}-60^{\circ}\right)$ to assess medial and lateral position and a long-axis view $\left(135^{\circ}-150^{\circ}\right)$ to monitor anterior-posterior clip adjustments (Fig. 10). With an intercommissural view on the left of the screen and a long-axis view on the right side of the screen, the interventionalist may efficiently move the clip in real time to an appropriate trajectory and position above the mitral

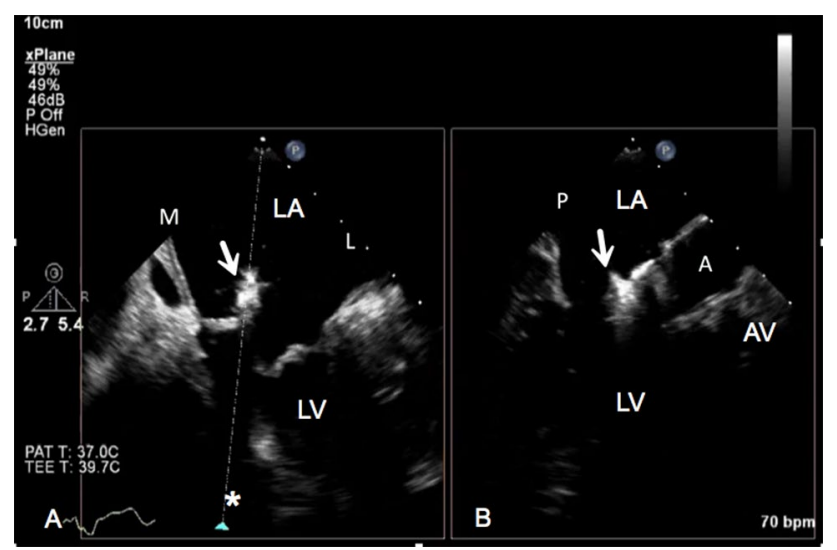

Fig. 10 Biplane advancement of clip delivery system (CDS). a CDS advanced in medial/lateral projection. Biplane angle (asterisk) tracks the CDS as it moves medial or lateral. $\mathbf{b}$ CDS in the anterior/posterior projection. Arrow clip, $M$ medial, $L$ lateral, $A$ anterior, $P$ posterior, $A V$ aortic valve, $L V$ left ventricle pathology and anticipated clip position. The echocardiographer must keep the CDS in view in both projections using iterative probe manipulations and management of the biplane angle in the intercommissural view. In correct clip position, no clip arms should be seen in the intercommissural view and both clip arms should be visualized in full length in the long-axis view. A single 3D TEE left atrium en face view allows real-time fine manipulation of the clip orientation so that it is positioned above the mitral valve pathology and perpendicular to the mitral valve coaptation line (Fig. 11).

\section{MitraClip positioning in the left ventricle}

The MitraClip system is advanced across the mitral valve into the left ventricle, just below the mitral valve. This movement is observed under biplane imaging; the intercommissural and long-axis views can be seen simultaneously to ensure that any rotation of the clip is identified. 3D en face TEE is imperative at this point to confirm position of the clip perpendicular to the coaptation line. The atrial 3D view is preferred to the ventricular view as it allows the interventionalist to make very subtle movements under the valve in an orientation that is the same as when the clip is above the valve. The $3 \mathrm{D}$ gain should be alternately reduced and increased in real time to remove and replace the leaflets above the clip. This ensures the perpendicular orientation of the clip relative to the leaflets (Fig. 12). If any changes in the clip position are necessary under the leaflets, they should be minimal to avoid entanglement in the chordae. If significant movements are necessary, the clip should be everted and withdrawn back into the left atrium where its orientation may be safely manipulated.

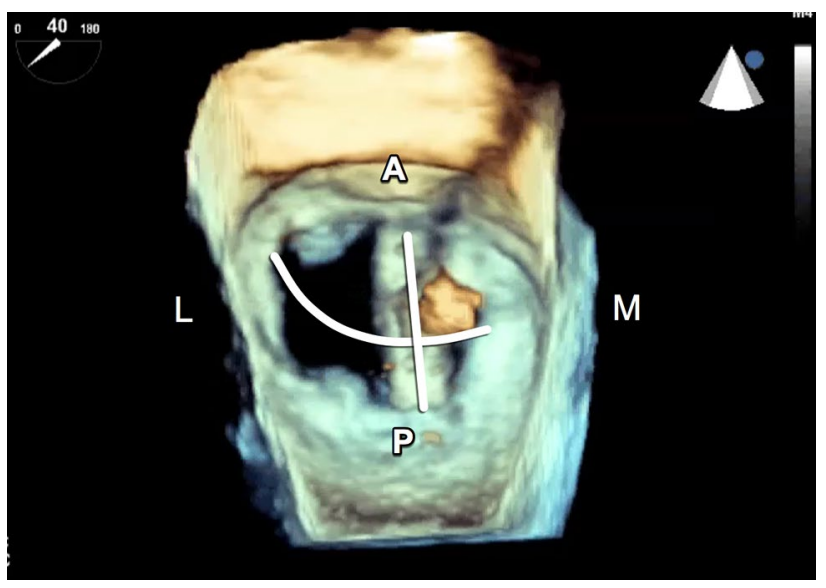

Fig. 11 En face view of the mitral valve with open clip above the mitral valve. The clip (straight line) is oriented perpendicular to the mitral valve coaptation line (curved line). This view allows the interventionalist to adjust the position or rotation of the clip safely in the atrium prior to crossing into the left ventricle for the grasp. $L$ lateral, $A$ anterior, $M$ medial, $P$ posterior 


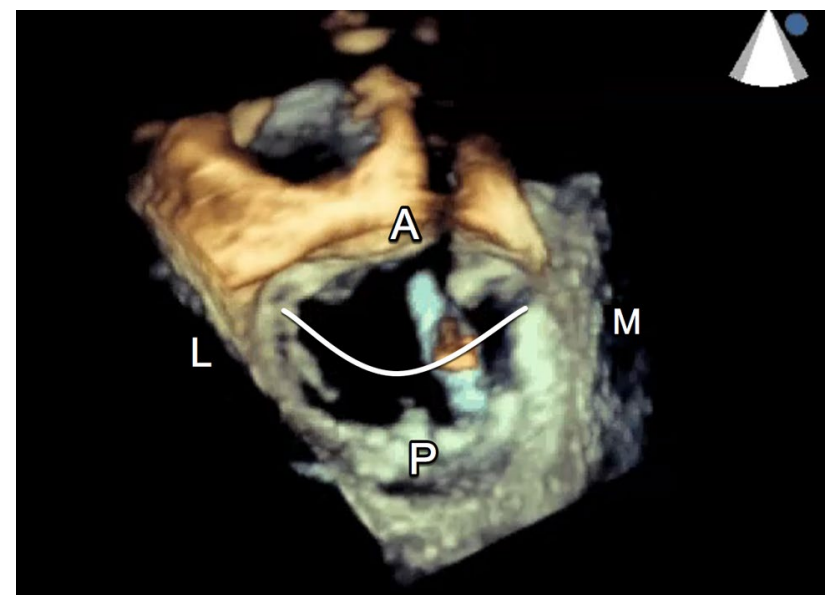

Fig. 12 En face view of the mitral valve with clip in the LV, located medially. The gain has been reduced to exclude the mitral valve leaflets but still display the clip arms. By alternating the gain, the perpendicularity of the clip to the coaptation line (curved white line) can be confirmed. $L$ lateral, $A$ anterior, $M$ medial, $P$ posterior

\section{Grasping the leaflets and verification of adequate leaflet insertion}

Once the clip is in adequate position under the mitral valve, the echocardiographer must move to the appropriate grasp TEE image. This image is created by positioning the probe and omniplane to visualize equal clip arm lengths, anterior and posterior leaflets and the shaft of the clip. The omniplane, just like the clip, is perpendicular to the coaptation line. The system is then slowly retracted towards the left atrium to allow the mitral leaflets to rest on the open clip arms. Once the leaflets are visualized over the clip arms and with tips ideally adjacent to the shaft, the grippers are lowered onto the leaflets (Fig. 13). Decreasing the sector width and lengthening the acquisition to obtain longer loop is helpful so that the grasp may be reevaluated during leaflet insertion assessment. Leaflet insertion should be verified in multiple angles-first at $0^{\circ}$ by advancing the TEE probe first to the anterior leaflet insertion and then to the posterior leaflet. The leaflets will drape over the clip arms with a reduction in leaflet mobility. An intercommissural view is then obtained with anterior and posterior rotation visualizing the leaflets draped over the clip arms. A long-axis/grasp view is then obtained to also confirm adequate insertion (Fig. 14). A 3D en face view should show a double-orifice valve with an adequate tissue bridge over the clip arms (Fig. 15). If these images confirm insertion, the clip may be tightened in the intercommissural view with color Doppler. Generally, if there is significantly improved coaptation, the mitral regurgitation will improve. After closure of the clip, insertion should be reconfirmed with TEE. Utilize continuous wave Doppler through the orifice to assess for an increased diastolic gradient. If the clip is in the appropriate position with adequate MR reduction and adequate grasp confirmed without stenosis, the clip may be released. If the MR jet is

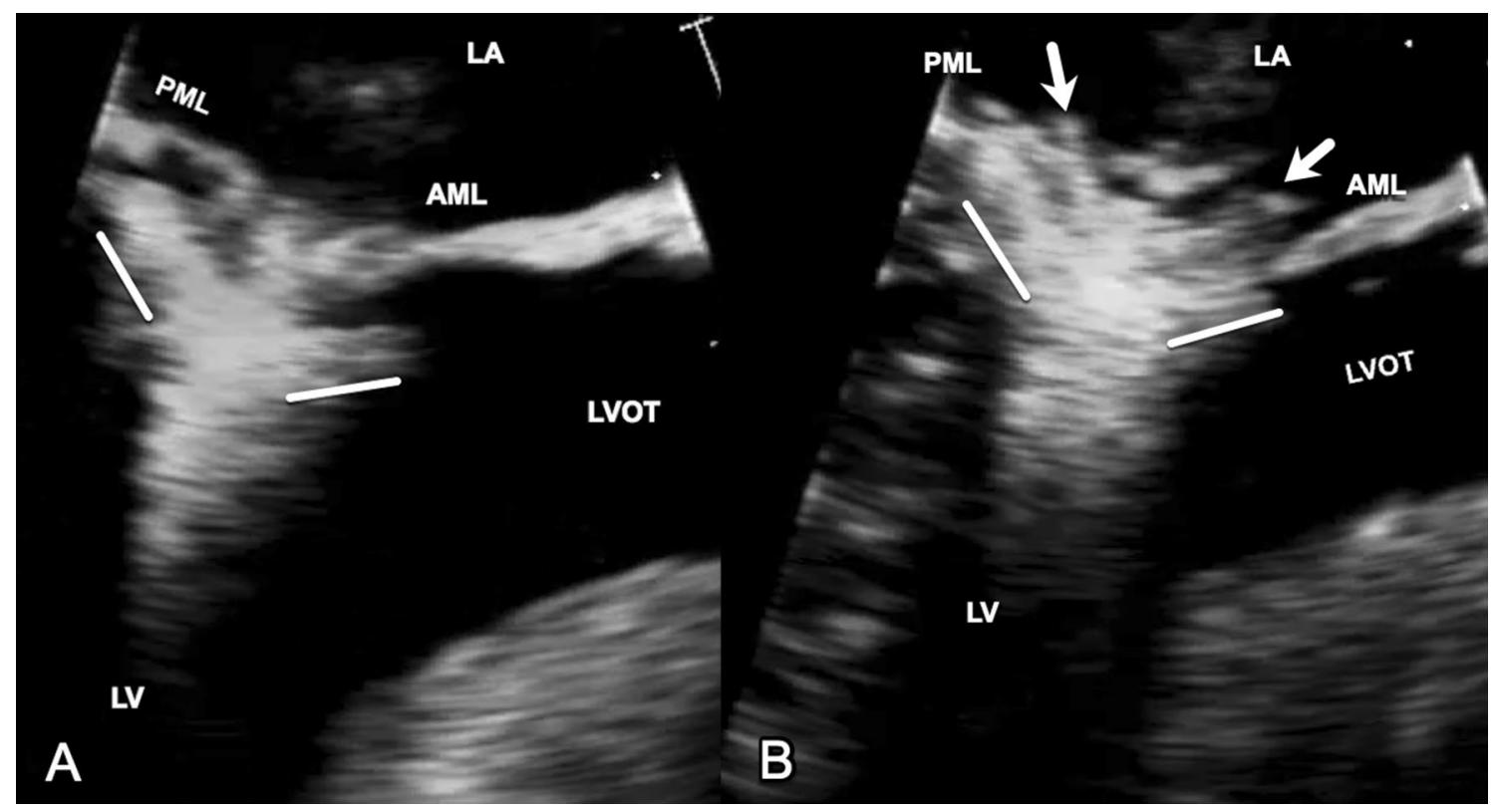

Fig. 13 Two-dimensional grasp view. a Prior to grasp, the omniplane and probe position are manipulated to visualize the leaflets, clip arms (solid white line) and shaft. b During the grasp, the leaflet is visualized being grasped between the clip arms (solid white line) and the frictional elements (arrows). $L A$ left atrium, $L V$ left ventricle, $P M L$ posterior mitral valve leaflet, $A M L$ anterior mitral leaflet, $L V O T$ left ventricular outflow tract 


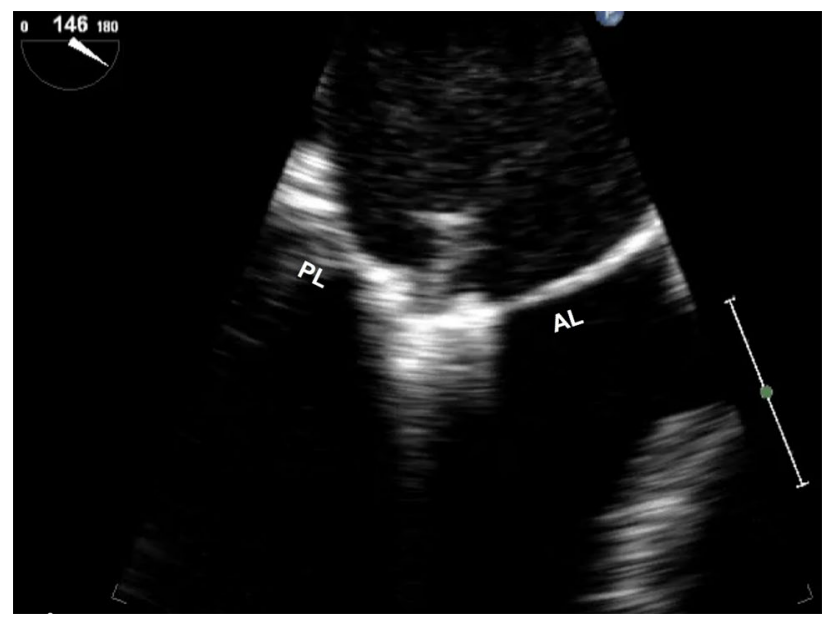

Fig. 14 Confirmation of grasp. Image at grasp angle demonstrates leaflets draped over the clip arms. Additional views are necessary to assess adequate grasp, including at $0^{\circ}$ and $3 \mathrm{D}$ en face. $P L$ posterior leaflet, $A L$ anterior leaflet

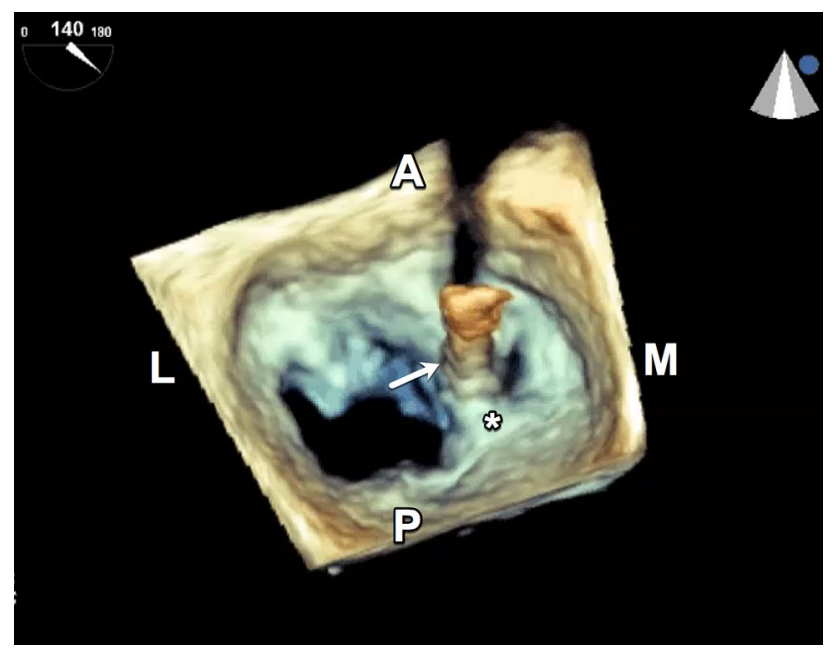

Fig. $153 \mathrm{D}$ en face view with tissue bridge draped over clip arms. Asterisk posterior leaflet tissue draped over clip arm, Arrow shaft of clip, $L$ lateral, $A$ anterior, $M$ medial, $P$ posterior

broad and two or three clips are necessary to reduce MR, then the first clip should be positioned at a location in the valve to allow for successful placement of the additional clips. Generally, it is easier to place the initial clip on the medial side of the MR jet to facilitate steering the subsequent clips lateral to the first clip. If the first clip is placed laterally, then the first clip may interact with the subsequent clips as they are steered into position.

After each clip is placed, a thorough assessment of mitral regurgitation with pulmonary vein assessment and transmitral pressure gradients should be performed. The diastolic transmitral gradient is assessed by continuous wave Doppler. A limitation is that Doppler measurements are highly influenced by heart rate, cardiac output, and residual mitral regurgitation. Nevertheless, transmitral pressure gradient assessed by continuous wave Doppler can be performed easily and is feasible and superior to direct peri-interventional assessment with mitral valve area [7]. It has been demonstrated that transmitral pressure gradient has the same validity in assessing double-orifice mitral valve as a single orifice mitral valve [8].

Planimetry of the mitral valve may be performed in short-axis transgastric views and with mid-esophageal 3D echocardiography [9]. The edges of the mitral valve leaflets should be easily seen. The inner edge of each orifice is then traced and the areas of each orifice are combined to calculate the total mitral valve area. The final geometry of the mitral valve and assessment of the newly formed orifices are best evaluated in $3 \mathrm{D}$ en face TEE views.

\section{After clip release}

Once the clip is released, the distal end of the CDS is the catheter shaft. This shaft may injure the left atrial wall if it encounters the wall as it is retracted into the SGC. Utilizing similar imaging angles as necessary to visualize the CDS into the left atrium, the CDS is imaged exiting the left atrium.

An iatrogenic atrial septal defect (iASD) will remain after the SGC is removed from the left atrium (Fig. 16). Assuming no additional damage to the septum and predominantly left to right flow, the defect is generally of no clinical significance.

\section{Complications}

TEE is utilized as part of the preintervention evaluation, to guide the MitraClip procedure and to identify complications that may occur during the procedure. Although the rate of complication is low, complications may be significant [10]. Continuous, careful assessment and open communication between the echocardiographer and the interventionalist will reduce the risk of complications. To reduce complications, the position and trajectory of all catheters, wires and delivery systems should be continuously imaged with TEE so the interventionalist can avoid cardiac injury. Careful alignment and meticulous imaging of clip positioning, advancement, grasp and insertion will reduce the risk of clip-specific complications such as chordae entanglement or single leaflet attachment. [5].

\section{Conclusions}

Edge-to-edge repair with the clip system is an echocardiography guided procedure. Success in safe MR reduction requires careful pre-procedure assessment and meticulous 


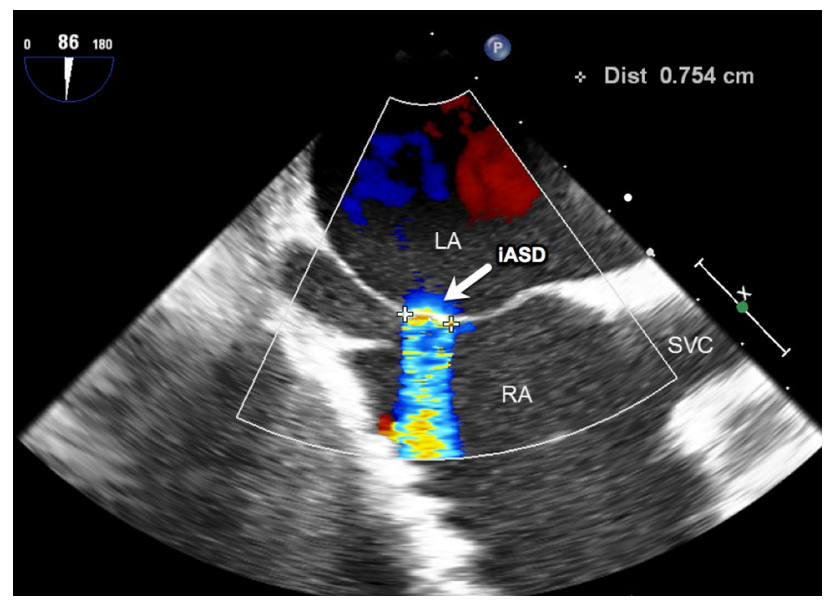

Fig. 16 Iatrogenic atrial septal defect (iASD) with left to right flow after removal of steerable guide catheter. $L A$ left atrium, $R A$ right atrium, SVC superior vena cava, iASD iatrogenic atrial septal defect

procedural imaging with frequent communication between the interventionalist and the echocardiographer. The team approach and imaging skills required to successfully guide this procedure will certainly translate into future transcatheter valve interventions.

\section{Compliance with ethical standards}

Conflict of interest Dr. Smith is a consultant for Millipede Medical and Pipeline. He is also a trainer for Abbott and provides imaging education for mitral valve interventions. He has stock in Millipede and receives honoraria and travel compensation from Abbott and Millipede. Dr. Aman has no conflicts of interest.

Open Access This article is distributed under the terms of the Creative Commons Attribution 4.0 International License (http://creativeco mmons.org/licenses/by/4.0/), which permits unrestricted use, distribution, and reproduction in any medium, provided you give appropriate credit to the original author(s) and the source, provide a link to the Creative Commons license, and indicate if changes were made.

\section{References}

1. Feldman T, Kar S, Elmariah S, et al. Randomized comparison of percutaneous repair and surgery for mitral regurgitation: 5-year results of everest II. J Am Coll Cardiol. 2015;66:2844-54.

2. Lim DS, Reynolds MR, Feldman T, et al. Improved functional status and quality of life in prohibitive surgical risk patients with degenerative mitral regurgitation after transcatheter mitral valve repair. J Am Coll Cardiol. 2014;64:182-92.

3. Stone GW, Lindenfeld J, Abraham WT, et al. Transcatheter mitral-valve repair in patients with heart failure. N Engl J Med. 2018;379(24):2307-18.

4. Alfieri O, Maisano F, De Bonis M, et al. The double-orifice technique in mitral valve repair: a simple solution for complex problems. J Thorac Cardiovasc Surg. 2001;122:674-81.

5. Nyman CB, Mackensen GB, Jelacic S, et al. Transcatheter mitral valve repair using the edge-to-edge clip. J Am Soc Echocardiogr. 2018;31:434-53.

6. Gripari P, Maffessanti F, Tamborini G, et al. Patients selection for MitraClip: time to move to transthoracic echocardiographic screening? Int J Cardiol. 2014;176:491-4.

7. Biaggi P, Felix C, Gruner C, et al. Assessment of mitral valve area during percutaneous mitral valve repair using the MitraClip system: comparison of different echocardiographic methods. Circ Cardiovasc Imaging. 2013;6:1032-40.

8. Maisano F, Redaelli A, Pennati G, et al. The hemodynamic effects of double-orifice valve repair for mitral regurgitation: a 3D computational model. Eur J Cardiothorac Surg. 1999;15:419-25.

9. Sürder D, Pedrazzini G, Gaemperli O, et al. Predictors for efficacy of percutaneous mitral valve repair using the MitraClip system: the results of the MitraSwiss registry. Heart. 2013;99:1034-40.

10. Maisano F, Franzen O, Baldus S, et al. Percutaneous mitral valve interventions in the real world: early and 1-year results from the ACCESS-EU, a prospective, multicenter, nonrandomized postapproval study of the MitraClip therapy in Europe. J Am Coll Cardiol. 2013;62:1052-61.

Publisher's Note Springer Nature remains neutral with regard to jurisdictional claims in published maps and institutional affiliations. 